\title{
GMR
}

\section{Iron excess in rice: from phenotypic changes to functional genomics of WRKY transcription factors}

\author{
V.E. Viana ${ }^{1,2}$, N. Marini ${ }^{1}$, T. Finatto ${ }^{3}$, I. Ezquer ${ }^{4}$, C. Busanello ${ }^{1}$, \\ R.S. dos Santos ${ }^{1}$, C. Pegoraro ${ }^{1}$, L. Colombo ${ }^{4}$ and A. Costa de Oliveira ${ }^{1}$ \\ ${ }^{1}$ Centro de Genômica e Fitomelhoramento, \\ Departamento de Fitotecnia, Faculdade de Agronomia Eliseu Maciel, \\ Universidade Federal de Pelotas, Pelotas, RS, Brasil \\ ${ }^{2}$ Centro de Desenvolvimento Tecnológico, \\ Universidade Federal de Pelotas, Pelotas, RS, Brasil \\ ${ }^{3}$ Universidade Tecnológica Federal do Paraná, \\ Campus Pato Branco, Pato Branco, PR, Brasil \\ ${ }^{4}$ Dipartimento di BioScienze, \\ Università Degli Studi di Milano, Milan, Italy \\ Corresponding author: A. Costa de Oliveira \\ E-mail: acostol@terra.com.br
}

Genet. Mol. Res. 16 (3): gmr16039694

Received April 6, 2017

Accepted July 3, 2017

Published September 27, 2017

DOI http://dx.doi.org/10.4238/gmr16039694

Copyright $(2017$ The Authors. This is an open-access article distributed under the terms of the Creative Commons Attribution ShareAlike (CC BY-SA) 4.0 License.

ABSTRACT. Iron (Fe) is an essential microelement for all living
organisms playing important roles in several metabolic reactions.
Rice (Oryza sativa L.) is commonly cultivated in paddy fields, where
Fe goes through a reduction reaction from $\mathrm{Fe}^{3+}$ to $\mathrm{Fe}^{2+}$. Since $\mathrm{Fe}^{2+}$ is
more soluble, it can reach toxic levels inside plant cells, constituting
an important target for studies. Here we aimed to verify morphological
changes of different rice genotypes focusing on deciphering the
underlying molecular network induced upon Fe excess treatments with
special emphasis on the role of four WRKY transcription factors. The
transcriptional response peak of these WRKY transcription factors in

Genetics and Molecular Research 16 (3): gmr16039694 
rice seedlings occurs at 4 days of exposition to iron excess. OsWRKY55like, OsWRKY46, OsWRKY64, and OsWRKY113 are up-regulated in BR IRGA 409, an iron-sensitive genotype, while in cultivars Nipponbare (moderately resistant) and EPAGRI 108 (resistant) the expression profiles of these transcription factors show similar behaviors. Here is also shown that some cis-regulatory elements known to be involved in other different stress responses can be linked to conditions of iron excess. Overall, here we support the role of WRKY transcription factors in iron stress tolerance with other important steps toward finding why some rice genotypes are more tolerant than others.

Key words: Abiotic stress; Gene regulation; Morphological adaptations; Iron homeostasis

\section{INTRODUCTION}

Adverse environmental conditions that hinder plant development are generally described as abiotic stresses. These conditions include drought, soil salinity, low temperature, radiation, and even excessive amounts of some nutrients (Banerjee and Roychoudhury, 2015). Copper, zinc, manganese, molybdenum, and iron (Fe) are essential elements that play important roles in redox reactions, and as part of several enzymes (Nagajyoti et al., 2010). Fe is required by many enzymes for proper biological function, playing an essential role in basic cellular processes. Present in electron transfer reactions during mitochondrial respiration and chloroplast photosynthesis, $\mathrm{Fe}$ is used as a cofactor in the form of Fe-S clusters or heme (Thomine and Vert, 2013). However, in excess, Fe is toxic and can produce reactive oxygen species (ROS) via Fenton reaction. These ROS can damage membranes, proteins, and DNA (Nagajyoti et al., 2010). In aerobic soils, Fe is found in its less soluble form $\left(\mathrm{Fe}^{3+}\right)$, but in waterlogged soils, $\mathrm{Fe}$ is reduced to its more soluble form $\left(\mathrm{Fe}^{2+}\right)$ due to the anaerobic condition, where $\mathrm{pH}$ is lower. The excessive $\mathrm{Fe}^{2+}$ uptake by plants causes some symptoms such as leaf bronzing, scanty and coarse roots (Pereira et al., 2013). Iron concentration in soil solution has been found to range from 10 to more than $2000 \mathrm{mg} / \mathrm{L}$ (Becker and Asch, 2005). However, it is suggested that $300 \mathrm{mg} / \mathrm{L}$ is the optimal concentration for screening tolerant genotypes (Dobermann and Fairhurst, 2000; Elec et al., 2013). It is estimated that Fe excess can cause yield losses of up to 400 $\mathrm{kg} / \mathrm{ha}$, losses that are strongly correlated with the symptom of leaf bronzing (Audebert and Fofana, 2009). Although this problem can cause deep consequences to the agricultural economy, little is known about the molecular mechanisms involved in the response to $\mathrm{Fe}$ excess (Ricachenevsky et al., 2010).

The study of transcription factors (TFs) is of great importance in improving plant stress tolerance, since these genes can regulate the expression of many others, resulting in deep physiological modifications (Banerjee and Roychoudhury, 2015). This regulation occurs through its binding to specific DNA sequences in the promoter regions of other genes that are then repressed or activated (Wang et al., 2015). When TFs bind to these specific sequences, called cis-acting elements, they initiate a signal cascade inducing different stress-related genes, where they can also regulate their own selves amplifying the signal (Banerjee and Roychoudhury, 2015). WRKY TFs are characterized by a highly conserved

Genetics and Molecular Research 16 (3): gmr16039694 
WRKY domain with ca. 60 amino acid residues containing the WRKYGQK sequence and a zinc finger motif C2H2- or C2HC-type (Wu et al., 2005). WRKY genes are classified according to their WRKY domains, which are determinant for their functional analysis ( $\mathrm{Wu}$ et al., 2005). WRKY TFs are one of the most important superfamilies involved in stress tolerance (Banerjee and Roychoudhury, 2015). Several WRKYs are involved in processes related to plant growth and development, synthesis of secondary metabolites, resistance towards multiple bacterial or fungal agents, and even tolerance to different abiotic stresses (Phukan et al., 2016). Considering such importance of WRKY factors, here we aimed to analyze morphological changes of rice genotypes and connect them to the expression profile of different WRKY genes in plants under Fe excess.

\section{MATERIAL AND METHODS}

\section{Plant material and growth conditions}

Rice seedlings (cv. Nipponbare, Epagri 108 and BR IRGA 409) were grown in pots containing a nutrient solution (Yoshida et al., 1976) with a photoperiod of $16 \mathrm{~h}$ and temperature of $\sim 25^{\circ} \mathrm{C}$ for 14 days. The nutrient solution was changed every week and the $\mathrm{pH}$ was adjusted to 4.5 every day. Subsequently, for iron excess treatment, the plants were transferred to pots containing nutrient solution supplemented with $1 \mathrm{mM}(300 \mathrm{mg} / \mathrm{L})$ ferrous sulfate $\left(\mathrm{FeSO}_{4} \cdot 7 \mathrm{H}_{2} \mathrm{O}\right)$ at $\mathrm{pH} 4.0$ (Elec et al., 2013). Plants used as control were also transferred on the 14th day to the nutrient solution at $\mathrm{pH} 4.5$. The experiment consisted of three replicates in a random design, where each replicate comprised 30 seedlings. Leaves and roots were collected at 4,8 , and 12 days after stress application and stored at $-80^{\circ} \mathrm{C}$ until total RNA extraction.

\section{Morphological analysis}

Plants were also analyzed morphologically for traits known to be involved in the response to iron stress at 4, 8, and 12 days after stress application. Traits measured included: shoot length (SL), root length (RL), coleoptile length (CL), leaf number (LN), root number $(\mathrm{RN})$, shoot dry mass (SDM), and root dry mass (RDM). For dry weight analysis, shoots and roots were kept in an oven $\left(80^{\circ} \mathrm{C}\right)$ for $24 \mathrm{~h}$ (Krzyzanowski et al., 1999).

\section{Statistical analysis}

Analysis of variance (ANOVA) was performed using the $\mathrm{SAS}^{\circledR} 9.3$ Software. Means were separated by the Tukey HSD (honestly significant difference) test with $\mathrm{P} \leq 0.05$. Means were compared by the Dunnet test with $\mathrm{P} \leq 0.05$. A Pearson correlation analysis was performed to check the association between gene expression and morphological traits with $\mathrm{P} \leq 0.05$.

\section{RNA extraction and cDNA synthesis}

Total RNA was extracted from $2 \mathrm{~g}$ fresh tissue following the protocol described by TRIzol $^{\mathrm{TM}}$ Reagent manual (Invitrogen ${ }^{\mathrm{TM}}$, Carlsbad, CA, USA). Samples were treated with 
DNAseI (Invitrogen ${ }^{\mathrm{TM}}$ ). The quantity of the RNA was assessed spectroscopically and the quality through agarose gel electrophoresis. Each sample was reverse-transcribed into cDNA using the commercial kit SuperScript ${ }^{\mathbb{R}}$ III First-Strand System for RT-PCR (Invitrogen ${ }^{\mathrm{TM}}$ ) from $2 \mu \mathrm{g}$ RNA.

\section{Real-time quantitative reverse transcription-PCR (RT-qPCR) analysis}

The RT-qPCR experiment was performed according to MIQE guidelines (Bustin et al., 2009), using oligonucleotide pairs for four WRKY up-regulated genes in rice shoots exposed to iron toxicity using a microarray approach (Finatto et al., 2015) (Table S1). Oligonucleotides were designed from sequences deposited in The Rice Annotation Project Data Base (RAPDB), using Primer Express ${ }^{\circledR}$ (Applied Biosystems ${ }^{\mathrm{TM}}$, Foster City, CA, USA) (Table S2). The criteria used for primer selection were amplicon size between 50 and $150 \mathrm{bp}$, CG content between 40 and $60 \%$, and melting temperature ranging from $60^{\circ}$ to $65^{\circ} \mathrm{C}$ according to Applied Biosystems ${ }^{\mathrm{TM}}$ recommendations. The RT-qPCR assay was conducted in triplicate in a Bio$\mathrm{Rad}^{\mathrm{TM}}$ iCycler iQ Optical System (software version 3.0a) using iQ SYBR Green Supermix (Bio-Rad ${ }^{\mathrm{TM}}$, Hercules, CA, USA). The quantification was performed according to the ${ }^{\Delta \Delta} \mathrm{Ct}$ method (Livak and Schmittgen, 2001), where elongation factor $\alpha(E F 1-\alpha)$ was used as an endogenous reference (Jain et al. 2006).

\section{Search of cis-regulatory elements (CRE)}

To compare CRE between japonica and indica subspecies, we analyzed WRKY promoters of Nipponbare, representing japonica, and WRKY promoters of 9311, representing indica. The putative promoters $(1.0 \mathrm{kbp}$ upstream of transcription start site) (De Bodt et al., 2006) of Nipponbare genes were obtained from RAP-DB (http://rapdb. dna.affrc.go.jp) while the sequences of 9311 genes were obtained from the EnsemblPlants database (http://plants.ensembl.org/index.html). The CRE information was obtained from PlantCare database (http://bioinformatics.psb.ugent.be/webtools/plantcare/html) (Lescot et al., 2002) and the analysis was performed considering just CRE with matrix score $\geq 5$ (Bazzini et al., 2009).

\section{RESULTS}

\section{Morphological analysis}

ANOVA showed significant effects (F-test; $\mathrm{P} \leq 0.05)$ for genotype and treatment (Table S3). There was also a significant interaction between these factors for LN and CL, determining that the variation due to treatment effect on the genotypes had different magnitudes.

It was possible to notice that Nipponbare, BR IRGA 409 (sensitive), and Epagri 108 (tolerant) showed greater SL and SDM when these were under iron excess (Figure 1). Regarding RL, none of the genotypes showed differences. Epagri 108 differed from the other two, with greater RN when stressed. The development of new roots could be a resistance mechanism since the older roots accumulated iron forming a ferric plaque, which prevented nutrient absorption. It is also possible to notice that control plants of every genotype had lower RDM (Figure 1).

Genetics and Molecular Research 16 (3): gmr16039694 



Root number

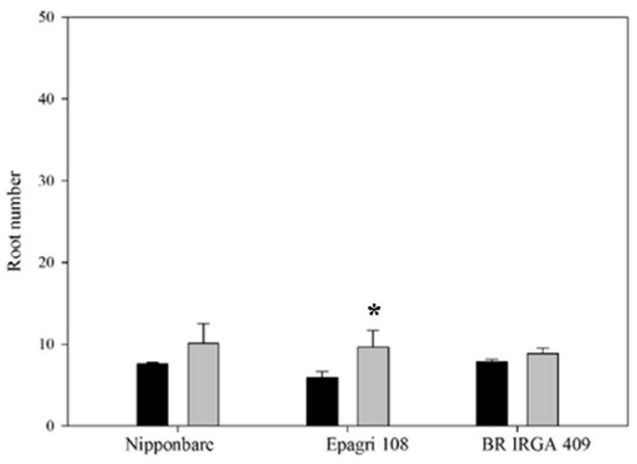

Shoot dry weight

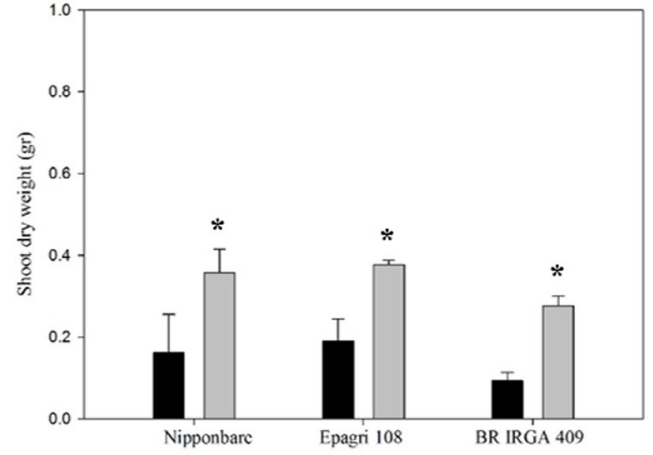

Root dry weight

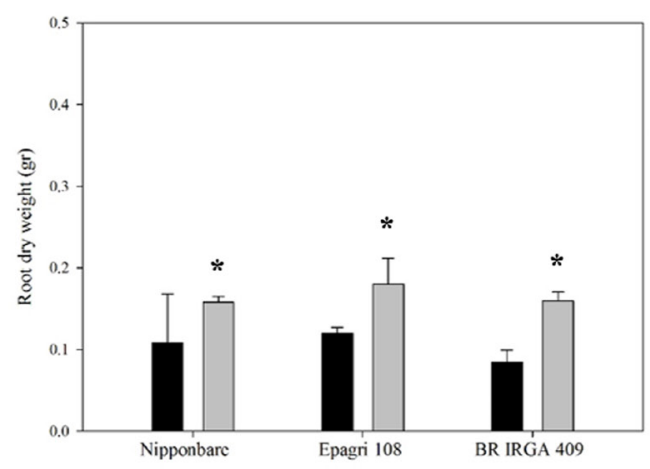

Control

12 days under iron excess

Figure 1. Analysis of shoot length, root length, root number, shoot dry weight, and root dry weight of rice seedlings (Nipponbare, BR IRGA 409, and EPAGRI 108), comparing control and stressed plants (12 days under iron excess). Means were compared to the control by the Dunnet test $(* \mathrm{P} \leq 0.05)$.

Genetics and Molecular Research 16 (3): gmr16039694 
A regression analysis for LN and CL showed that EPAGRI 108 increased its LN (Figure 2 ), suggesting continued growth during stress. However, Nipponbare was more sensitive, with a decrease in this trait (Figure 2).
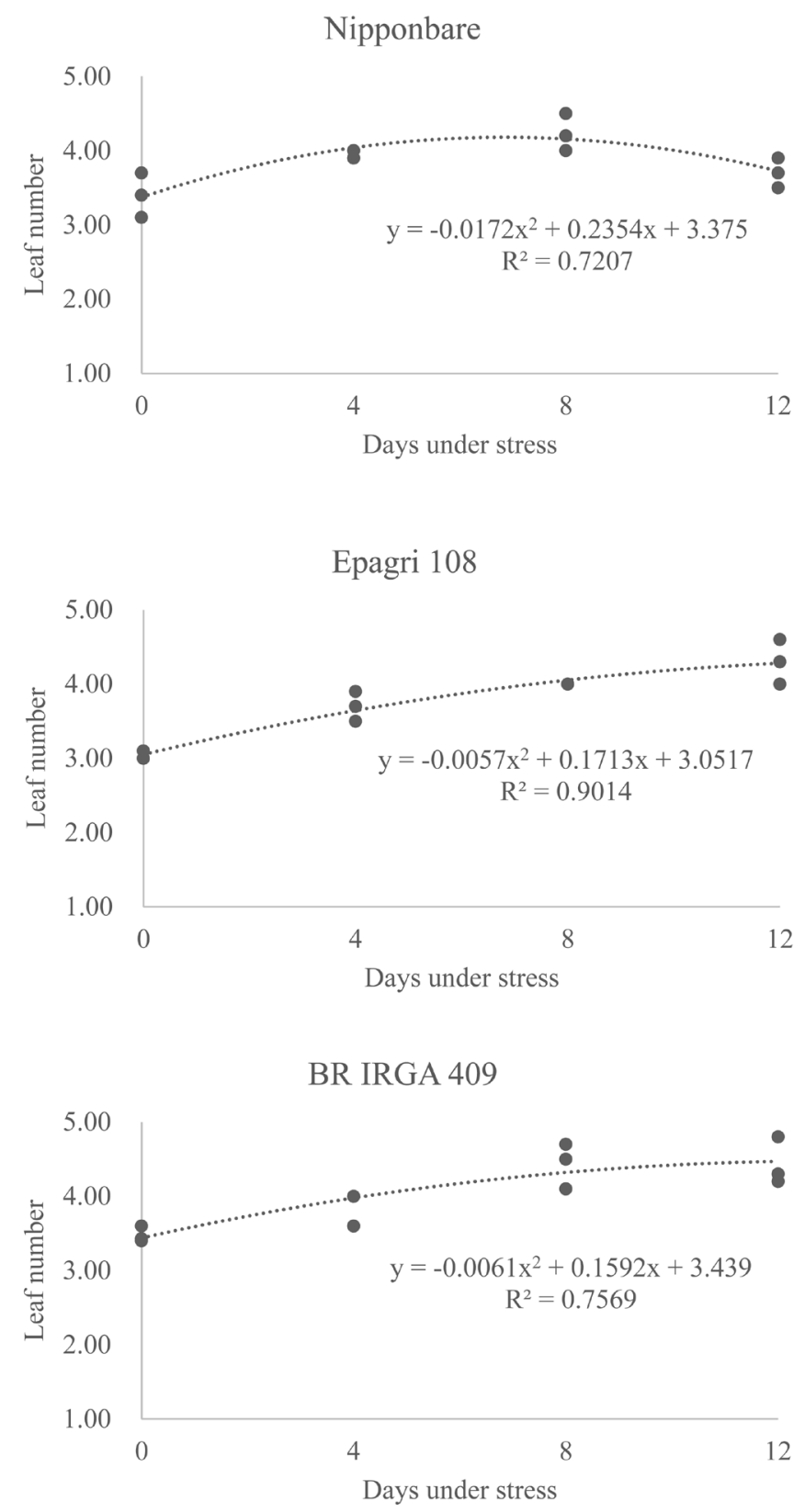

Figure 2. Regression analysis for leaf number of rice seedlings (Nipponbare, EPAGRI 108, and BR IRGA 409), analyzed at $0,4,8$, and 12 days under iron excess.

Genetics and Molecular Research 16 (3): gmr16039694 
Significant differences regarding CL were found when comparing genotypes, where Nipponbare showed a progressive decrease during stress (Figure 3). Thus, while in control conditions, Nipponbare showed longer coleoptiles when compared to BR IRGA 409 and Epagri 108, the same did not occur at 12 days under stress (DUS) (Figure 3).

BR IRGA 409 showed a strong decrease in CL at 8 DUS. Coleoptile was involved in stress tolerance, and shorter CL found in this cultivar was another indication of the inability of this genotype to tolerate such stress (Figure 3). EPAGRI 108 did not show CL variations during the exposure to iron stress, supporting its classification as a tolerant genotype (Figure 3).
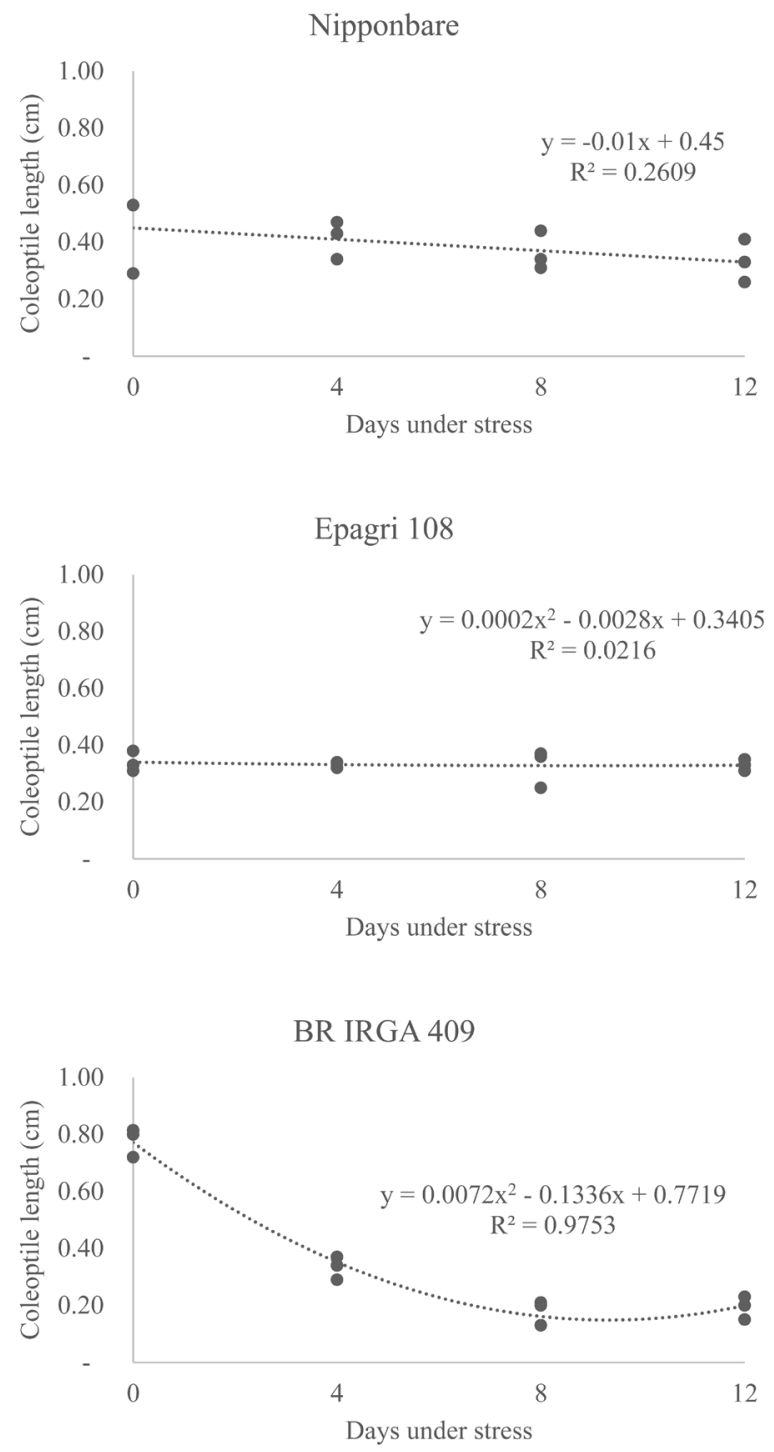

Figure 3. Regression analysis for coleoptile length of rice seedlings (Nipponbare, EPAGRI 108, and BR IRGA 409), analyzed at $0,4,8$, and 12 days under iron excess.

Genetics and Molecular Research 16 (3): gmr16039694 


\section{Relative expression level (REL) analysis}

The expression profiles of four rice WRKYs in different vegetative tissues indicated that these genes responded to iron excess in both shoot and root (Figures 4 and 5). Roots that were the first tissues to make contact with iron responded by accumulating higher levels of transcription. In general, 4 DUS was enough to demonstrate the involvement of these genes in stress response, particularly for the sensitive BR IRGA 409. Distinct behaviors could be seen in each genotype and each tissue. In shoot tissues, Epagri 108 and Nipponbare were similar with little changes in transcript abundance, while BR IRGA 409 stood out, with more expressive changes (Figure 4). Regarding root tissues, Epagri 108 and BR IRGA 409 were similar, with expressive changes in transcript abundance, different from Nipponbare (Figure 5).

The REL of OsWRKY55-like, in shoot tissues, showed a 50- fold increase in BR IRGA 409 at 4 DUS, constituting the highest transcript accumulation of all. These data evidenced the involvement of OsWRKY55-like in early iron stress response. Still, the maintenance of this stress promoted a gradual decrease in transcript accumulation only in this sensitive genotype (Figure 4).

A similar situation was observed in shoots for OsWRKY46, but here the transcriptional response at 4 DUS was not higher than 5-fold for BR IRGA 409. However, it was significantly different from the other treatments, evidencing the involvement of OsWRKY46 in stress response (Figure 4).

Changes in shoot transcript abundance were also observed for OsWRKY64, especially in BR IRGA 409, which was already significantly higher at 4 DUS and reached a peak of 9-fold at 8 DUS. EPAGRI 108 and Nipponbare showed very mild changes (Figure 4).

Regarding shoot, for OsWRKY113 REL, the only change that could be observed was a small increase in BR IRGA 409 at 4 DUS (Figure 4).
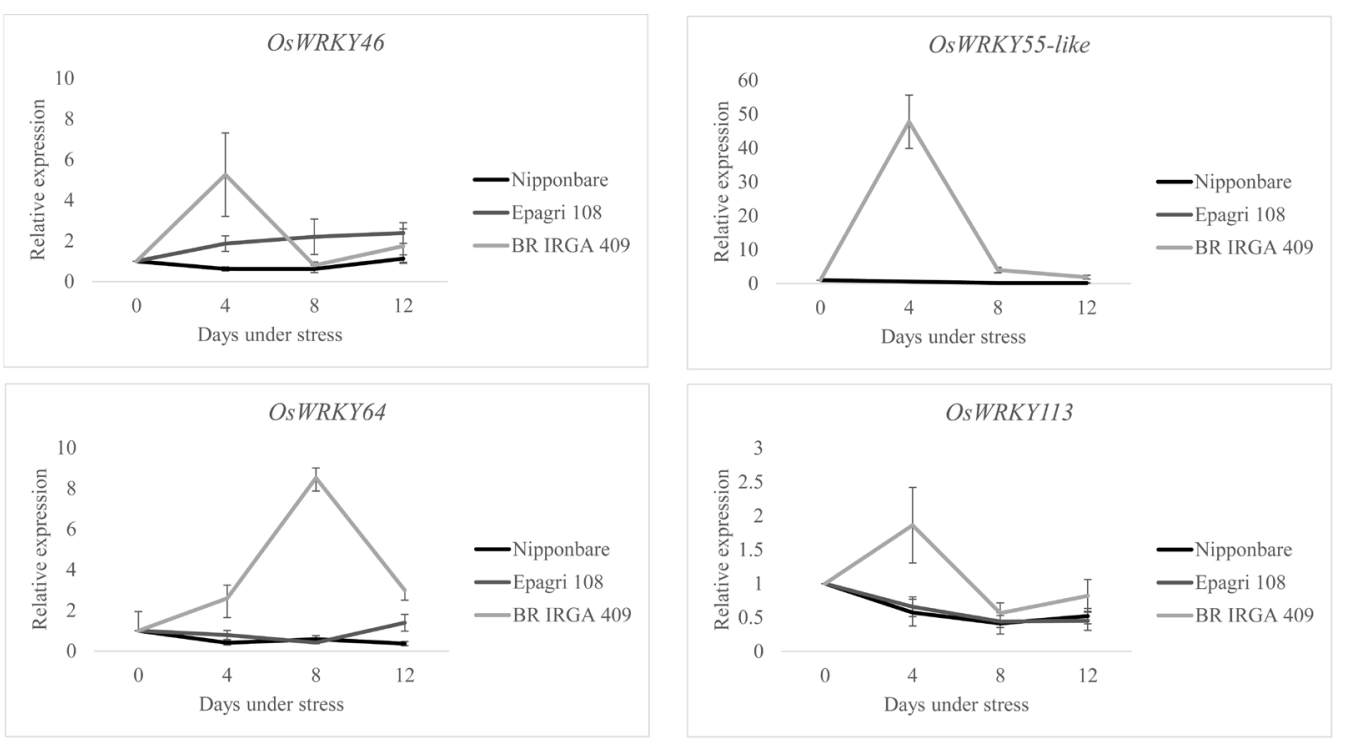

Figure 4. Relative expression levels of WRKY genes in shoot tissue of Nipponbare, EPAGRI 108, and BR IRGA 409 at $0,4,8$, and 12 days under iron stress.

Genetics and Molecular Research 16 (3): gmr16039694 
Roots showed higher sensitivity to stress when compared to shoot tissues (Figures 4 and 5). A significant increase in transcript abundance of OsWRKY55-like could be observed, where BR IRGA 409 and EPAGRI 108 showed 80- and 40-fold changes, respectively, when compared to the control condition (Figure 5). In the same way, an increase of 60- and 25-fold in the expression of OsWRKY46 was observed at 4 DUS in the sensitive and resistant genotypes, respectively (Figure 5). OsWRKY64 showed a significant increase in transcript abundance, around 60- and 65-fold in the sensitive and tolerant genotypes, respectively (Figure 5). Concerning to $O s W R K Y 113$, an increase of 25 - and 15-fold in sensitive and tolerant genotypes was detected, respectively (Figure 5). Considering these indications, it could be suggested that OsWRKY46, 64, 113, and OsWRKY55-like were involved in iron stress response in both root and shoot tissues.
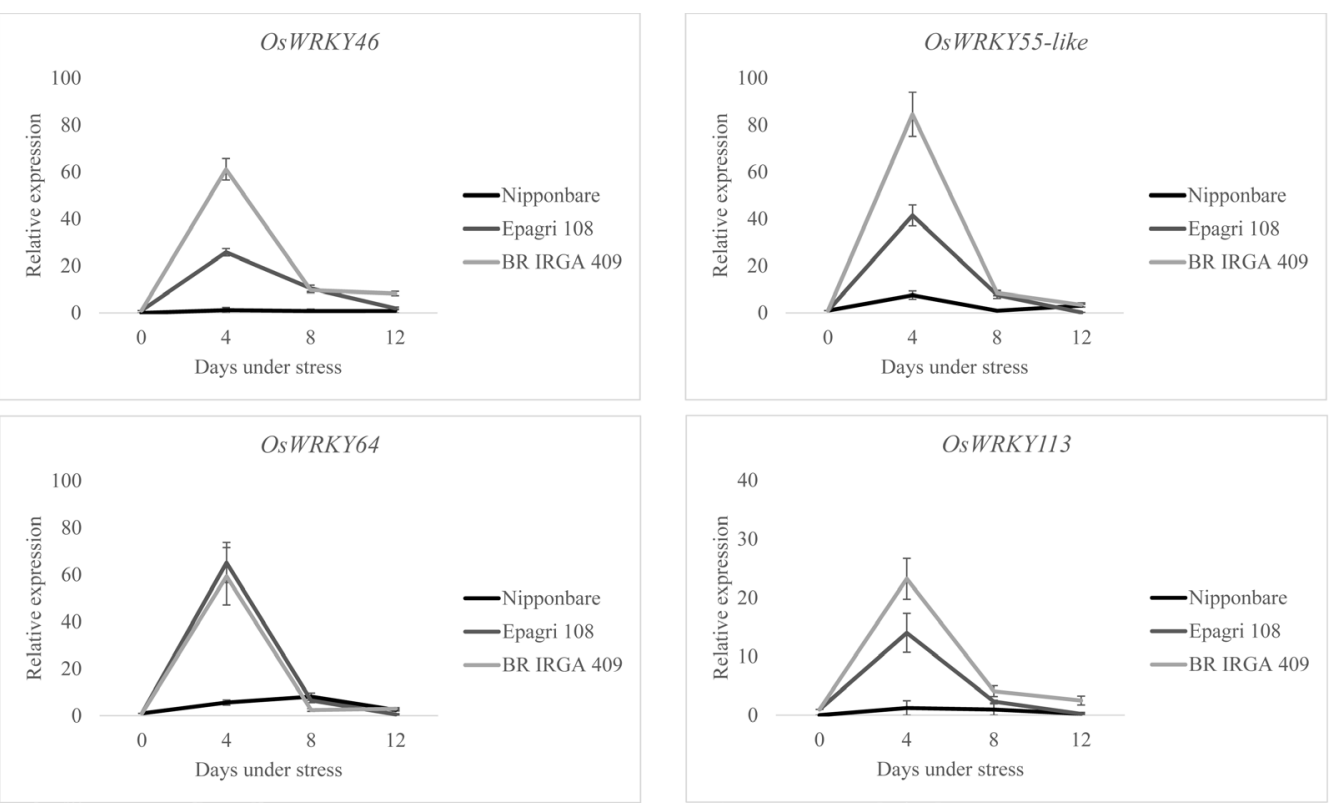

Figure 5. Relative expression of WRKY genes in root tissues of Nipponbare, EPAGRI 108, and BR IRGA 409 at $0,4,8$, and 12 days under iron stress.

\section{Effects of WRKY expression on morphological traits}

The correlation analysis enabled the understanding of how WRKY expression peaks could impact each morphological trait (Table 1). A positive correlation between OsWRKY46 expression at 4 DUS and SL at 12 DUS for Nipponbare suggested an effect of OsWRKY46 in promoting an increase in SL. This effect was larger than the one observed for the tolerant genotype EPAGRI 108, demonstrating that iron excess $(1 \mathrm{mM})$ did not affect the shoot growth in Nipponbare.

A positive correlation between OsWRKY113 REL and SL for EPAGRI 108 was observed. It differed from the observed for the sensitive genotype BR IRGA 409.

Genetics and Molecular Research 16 (3): gmr16039694 
Table 1. Pearson correlation analysis between gene expression of rice WRKYs (at 4 days under iron toxicity) and morphological traits (at 12 days under iron toxicity).

\begin{tabular}{|c|c|c|c|c|}
\hline \multicolumn{5}{|c|}{ Nipponbare } \\
\hline & OsWRKY55-like & OsWRKY46 & OsWRKY64 & OsWRKY113 \\
\hline SL & 0.8052 & $0.9969^{*}$ & 0.7818 & 0.7818 \\
\hline LN & -0.1121 & 0.4293 & \begin{tabular}{|l|}
-0.1502 \\
\end{tabular} & -0.1502 \\
\hline CL & 0.96 & 0.9632 & 0.9485 & 0.9485 \\
\hline SDM & 0.4465 & 0.8515 & 0.4118 & 0.4118 \\
\hline \multicolumn{5}{|c|}{ Epagri 108} \\
\hline $\mathrm{SL}$ & -0.8303 & 0.8499 & 0.8989 & $0.9985^{*}$ \\
\hline LN & -0.5831 & 0.6121 & 0.6891 & 0.9164 \\
\hline $\mathrm{CL}$ & $0.9989^{*}$ & -0.9965 & -0.9829 & -0.8343 \\
\hline SDM & 0.5899 & \begin{tabular}{|l|}
-0.5603 \\
\end{tabular} & \begin{tabular}{|l|}
-0.4735 \\
\end{tabular} & \begin{tabular}{|l|}
-0.0943 \\
\end{tabular} \\
\hline \multicolumn{5}{|c|}{ BR IRGA 409} \\
\hline SL & 0.8917 & -0.1386 & \begin{tabular}{|l|}
-0.4527 \\
\end{tabular} & 0.0538 \\
\hline LN & 0.6312 & 0.9386 & \begin{tabular}{|l|l|}
0.7757 \\
\end{tabular} & 0.9873 \\
\hline $\mathrm{CL}$ & 0.0196 & -0.9393 & \begin{tabular}{|l}
$-0.9998^{*}$ \\
\end{tabular} & -0.8561 \\
\hline SDM & 0.5643 & 0.9641 & \begin{tabular}{|l|}
0.8256 \\
\end{tabular} & $0.9971^{*}$ \\
\hline \multicolumn{5}{|c|}{ Nipponbare } \\
\hline $\mathrm{RL}$ & $-0.9996 *$ & \begin{tabular}{|l|l|}
$-0.9985^{*}$ \\
\end{tabular} & \begin{tabular}{|l|}
-0.8285 \\
\end{tabular} & 0.6135 \\
\hline $\mathrm{RN}$ & 0.6974 & 0.6794 & 0.2055 & $\begin{array}{l}-0.9901 \\
\end{array}$ \\
\hline RDM & -0.2016 & \begin{tabular}{|l|}
-0.1772 \\
\end{tabular} & 0.354 & 0.9098 \\
\hline \multicolumn{5}{|c|}{ Epagri 108} \\
\hline $\mathrm{RL}$ & -0.5579 & \begin{tabular}{|l|}
-0.7914 \\
\end{tabular} & 0.5121 & 0.0951 \\
\hline RN & -0.7539 & \begin{tabular}{|l|}
-0.9228 \\
\end{tabular} & 0.272 & -0.1662 \\
\hline RDM & 0.5692 & 0.2804 & 0.9865 & 0.9616 \\
\hline \multicolumn{5}{|c|}{ BR IRGA 409} \\
\hline $\mathrm{RL}$ & 0.1265 & 0.9206 & $\mid 0.9999 *$ & -0.7169 \\
\hline RN & 0.9899 & 0.6211 & 0.2805 & -0.8626 \\
\hline RDM & -0.9242 & -0.1356 & 0.2472 & 0.485 \\
\hline
\end{tabular}

*Significant correlation $(\mathrm{P} \leq 0.05)$. SL: shoot length; $\mathrm{LN}$ : leaf number; $\mathrm{CL}$ : coleoptile length; SDM: shoot dry mass; $\mathrm{RL}$ : root length; RN: root number; RDM: root dry mass.

A negative correlation was detected between OsWRKY64 expression and CL for BR IRGA 409, where an increase of 8.5-fold of OsWRKY64 REL led to shorter CL. This finding suggested that this genotype cannot maintain normal development of coleoptile under iron excess. The 1.8-fold increase of OsWRKY113 in BR IRGA 409 was positively correlated with SDM, suggesting that this gene can help maintaining biomass fixation.

In the Nipponbare background, OsWRKY55-like increased its expression in 7.5-fold, being negatively correlated with RL. A similar result was observed for OsWRKY46, which also had a 10-fold REL increase and was also negatively correlated with RL. At 12 DUS, Nipponbare showed a small increase of RL but, differently from the sensitive genotype, maintained its basal development.

\section{Identification of CRE in WRKY promoters}

Predicted CRE in the 1-kb upstream regions of Nipponbare and 9311 ranged from 1 to 44 and from 1 to 43 , respectively (Table S4). The total amount of CRE in each promoter was 17 in the OsWRKY55-like promoter of Nipponbare and 19 in the 9311 promoters. The OsWRKY46 promoter region showed 17 CRE in Nipponbare and 16 in 9311. For OsWRKY64, 16 and 14 CRE were found in Nipponbare and 9311, respectively, while for OsWRKY113, 19 different CRE were found in both genotypes (Figure 6 and Table S4). Although many of the CRE found here are known to be involved in different stress responses, none of these has ever been reported as being linked to conditions of iron excess. Venn diagrams illustrated 
intersections among these promoters for both genotypes, allowing us to detect the number of CRE that were shared between the upstream regions of these four genes (Figure 6 and Table S4).

Nipponbare

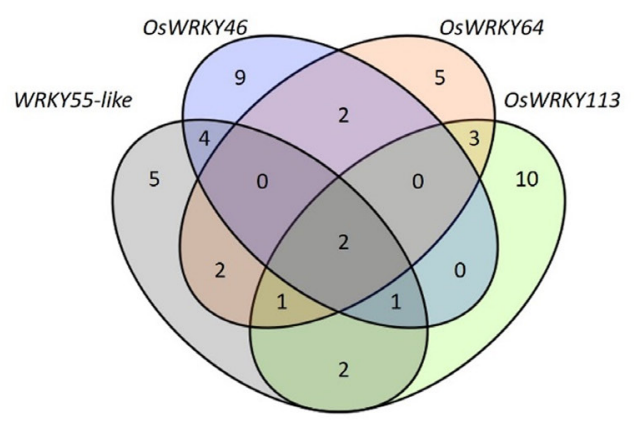

9311

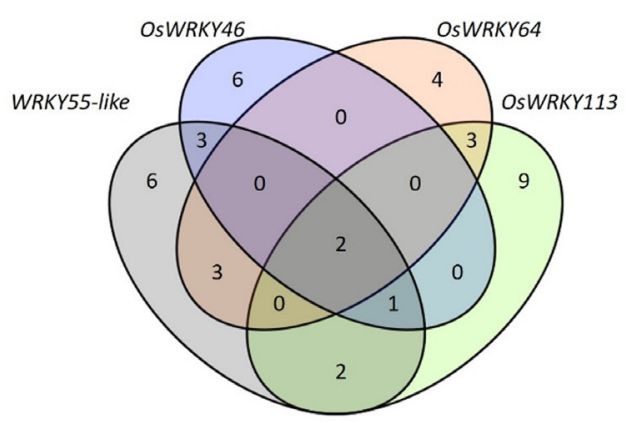

Figure 6. Venn diagram of the cis-regulatory elements shared in promoters (1-kb upstream) of WRKY genes in Nipponbare and 9311.

In Nipponbare, OsWRKY55-like and OsWRKY64 shared 70\% of their CRE while OsWRKY46 and OsWRKY113 shared 50\% (Figure 6). For 9311, a similar result was found where OsWRKY55-like and OsWRKY64 shared 65\%, while OsWRKY46 and OsWRKY113 shared 50\% of their CRE. These shared CRE could explain their similar regulatory behavior. G-box and TCAelement found in OsWRKY55-like, OsWRKY46, and OsWRKY113 for both genotypes (Table S4), also constituted interesting information, since the first CRE was known to be involved in light response while the second was involved in salicylic acid (SA) response, showing that the genetic regulation of rice under iron excess could share similar paths of response to these two factors. SP1, a light-responsive element, was found in OsWRKY55-like, OsWRKY46, and OsWRKY64 of 9311, but only in OsWRKY55-like and OsWRKY46 promoters of Nipponbare.

Some CRE were found only in one of the genotypes, this was the case of ACE, Box I, CE3, HSE, I-box, TACTC-box, which could be seen only in Nipponbare and were characterized to be involved in ABA, light, heat shock, and gibberellin responses.

\section{DISCUSSION}

To keep its homeostasis and avoid excessive Fe accumulation in leaves, plants have the ability to reduce Fe translocation to aerial parts, leading to an accumulation of Fe in roots (da Silveira et al., 2007). The presence of high iron concentrations in root symplasts of EPAGRI 108 (7 mg/g RDM compared to BR IRGA 409 with $1.93 \mathrm{mg} / \mathrm{g}$ RDM) plants under iron excess, indicates its ability to deal with higher intracellular concentrations of iron at the root zone, ability which plays an important role in determining its tolerance, protecting shoots from high iron concentrations (Stein et al., 2014). Thus, a second strategy is well proposed where a mechanism of iron oxidation on root surface can be used by plants to tolerate iron excess (Becker and Asch, 2005). Iron oxidation on root surface can occur through its precipitation $\mathrm{asFe}^{3+}$, caused by the oxygen carried from shoots to roots allowing plants to tolerate this stress due to the exclusion of iron from plant cells (Nava and Bohnen, 2002). Plants with iron excluder (that oxidize and retain iron) can develop aerenchyma and a large number of lateral roots (Wu et al., 2014).

Genetics and Molecular Research 16 (3): gmr16039694 
BR IRGA 409 and Epagri 108 have both iron oxidation ability, suggesting that this ability is not sufficient to explain their different sensitivity to iron (Nava and Bohnen, 2002). Even though RDM was significantly higher in every genotype after 12 DUS, RL was not different from control and only EPAGRI 108 presented higher RN. The results of RDM and, in EPAGRI 108, RN might be linked to the strategy of iron oxidation that created a plaque of iron on the root surface of plants at 12 DUS, generating the need for new root emission and suggested that Epagri 108 can act as an excluder.

A significant difference between genotypes was found for SDM and RDM. Nipponbare and BR IRGA 409 showed lower RDM when compared to the tolerant genotype EPAGRI 108. Previous results from our group detected similar leaf iron accumulation levels for Nipponbare and BR IRGA 409 at 3 DUS (2000 mg/L FeSO $\left..7 \mathrm{H}_{2} \mathrm{O}\right)$, finding 20,000 and 15,000 mg/kg for Nipponbare and BR IRGA 409, respectively (Bresolin and Costa de Oliveira, unpublished data).

Regarding $W R K Y$ transcriptional expression, we showed that the four genes analyzed here were responsive to iron excess (Figures 4 and 5). This is the third report linking WRKYS with iron excess response (Ricachenevsky et al., 2010; Finatto et al., 2015). The first shows that OsWRKY80, which is also involved in rice drought response and senescence, increases its transcriptional expression (3-fold) after 6 DUS at 500 ppm (Ricachenevsky et al., 2010). The second is a microarray analysis showing that 19 WRKYs are up-regulated when rice is under iron excess ( $7 \mathrm{mM}$ for 18 DUS), suggesting that this family can perform an important role in iron homeostasis (Finatto et al., 2015).

The identification of responsive TFs is an important step in the understanding of tolerance mechanisms since these genes can regulate the expression of many others, causing deep modifications in plant physiology. Genes involved in regulation of iron uptake and storage have been widely studied, especially under iron deficiency (Walker and Connolly, 2008). In Arabidopsis (Arabidopsis thaliana L.), many reports have linked iron deficiency and AtWRKY46 expression, suggesting its involvement in response to Fe deficiency. A phylogenetic tree of WRKY genes from rice and Arabidopsis demonstrates that AtWRKY46 and OsWRKY64 are both parts of group IIIa (Wu et al., 2005), suggesting that these should have similar roles. Also, wrky46-1 and wrky46-2 loss-of-function mutants have significantly affected Fe translocation from root to shoot. The inability of these genes in performing their roles properly causes chlorosis of new leaves via direct regulation of the iron transporter VITL1 (Vacuolar Iron Transporter1like1) transcript levels (Yan et al., 2016). In the tolerant EPAGRI 108, as well as Nipponbare, OsWRKY64 is less expressed in shoot tissue than it is in BR IRGA 409 after 12 DUS. The consequent lower expression of VITL1 in this cultivar, decreasing transport rate to leaves, may be one of the factors that lead to a better performance of this genotype in this stressful condition.

Although WRKYs are frequently related to biotic stress, little is known about the regulation of these genes in plants under iron excess. OsWRKY64 is known to be involved in transcriptional activation of genes in response to the rice pathogens Magnaporthe oryzae and Xanthomonas oryzae pv. oryza (Xoo) (Jimmy and Babu, 2015), while overexpression of OsWRKY31 can enhance resistance to fungal blast (Magnaporthe grisea) (Zhang et al., 2008). OsWRKY55 is also named as OsWRKY31 according to the nomenclature of the Rice WRKY Working Group (2012). Overexpression of OsWRKY31 (OsWRKY55) not only confers resistance to $M$. grisea but also causes a reduction of lateral root formation and elongation, suggesting that this gene should be an important component of signal transduction pathways of auxin and defense response in rice (Zhang et al., 2008). OsWRKY55, as well as OsWRKY45 and $O s P O X 22.3$, is involved in the response to benzothiadiazole treatment. Benzothiadiazole

Genetics and Molecular Research 16 (3): gmr16039694 
is an activator that can prime SA signaling pathway and consequently induce systemic acquired resistance. Thus, OsWRKY55 has an important role in plant defense mechanisms linked to SA (Baldoni et al., 2013).

SA is known to be involved in iron deficiency responses. The Arabidopsis pad4 (phytoalexin deficient 4) mutant shows a higher concentration of soluble iron when compared to wild-type plants when these are under iron deficiency. Since this gene is involved in SA biosynthesis, wild-type plants present SA accumulation while in the mutant this process is blocked. This suggests that increase in SA concentration induced by iron deficiency can activate iron translocation via transcriptional regulation of bHLH38/39 under downstream genes by signaling of auxin and ethylene. Both hormones were high concentrated by the increase of SA (Shen et al., 2016).

Regarding promoter regulation analysis, none of the CRE found in the upstream region of Nipponbare WRKYs is known to be involved in iron excess (Figure 7), but in other abiotic stress responses (Table S4). A precise promoter analysis identified an iron-dependent CRE that represses the expression of the maize (Zea mays L.) and Arabidopsis ferritin genes, ZmFerl and AtFer1, under iron excess (Petit et al., 2001). Another CRE was found to be involved in iron deficiency in the IDS2 gene (Fe-deficiency-specific clone No. 2) of barley (Hordeum vulgare L.). A transgenic approach in tobacco (Nicotiana tabacum L.) identified, in IDS2, the cis-acting iron deficiency-responsive elements 1 and 2 (IDE1/IDE2), the first CRE that are now known to be involved in the response to micronutrient deficiency in plants (Kobayashi et al., 2003). IDE1 and IDE2 induce iron deficiency response expression in roots of tobacco and in leaves and roots of rice, allowing the binding process of TF IDE-binding factor 1 and 2 (IDEF1/IDEF2) (Kobayashi and Nishizawa, 2012).

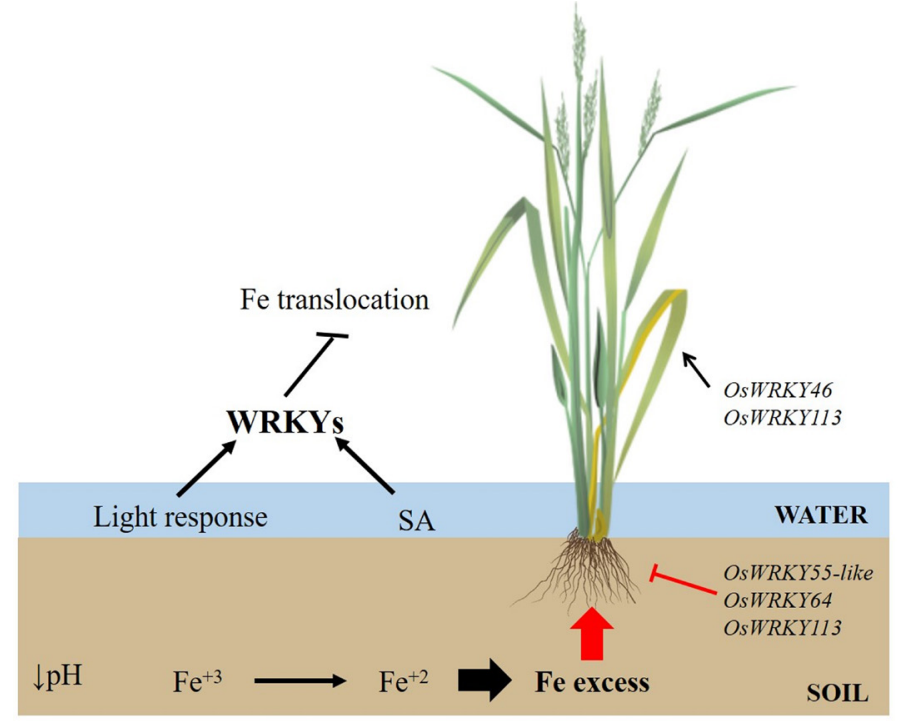

Figure 7. Model of the WRKY-mediated response to an iron excess in rice plants. In waterlogged soils with low redox potential and acidic $\mathrm{pH}$, the insoluble $\mathrm{Fe}^{3+}$ is reduced to soluble $\mathrm{Fe}^{2+}$, which then accumulates at higher magnitudes in plant tissues. To avoid Fe translocation from roots to leaves, WRKY transcription factors are activated. In root tissue, OsWRKY55-like, OsWRKY64, and OsWRKY113 can act to prevent its growth, while OsWRKY46 and OsWRKY113 promote the development of leaves. SA, salicylic acid. 
In summary, this study describes the pattern of expression of four iron-responsive WRKY genes and their association with different morphological traits. Here it is proposed that to avoid iron stress these WRKY factors may repress iron translocation to shoots with a peak of response at 4 DUS. Here OsWRKY55-like, OsWRKY64, and OsWRKY113 probably act regulating root elongation, repressing it under iron excess. Also, OsWRKY46 and OsWRKY113 should act in shoot tissue promoting its growth. The CRE analysis provides evidence that the molecular signaling involved in this response shares similarities with those mechanisms involved in light responses and SA pathway. The functional characterization of OsWRKY55like, OsWRKY46, OsWRKY64, and OsWRKY113 should clarify our understanding of iron toxicity tolerance mechanisms, making it possible to manipulate some of the regulatory networks controlled by these TFs.

\section{Conflicts of interest}

The authors declare no conflict of interest.

\section{ACKNOWLEDGMENTS}

Research supported by Coordenação de Aperfeiçoamento de Pessoal de Nível Superior (CAPES), Fundação de Amparo à Pesquisa do Estado do Rio Grande do Sul (FAPERGS), and Conselho Nacional de Desenvolvimento Científico e Tecnológico (CNPq). The EVO-CODE project, the IEF-METMADS project, the EXPOSEED-RISE project, and the Università degli Studi di Milano (Ass. ricerca Tipo A-2015, RTD-A) are gratefully acknowledged.

\section{REFERENCES}

Audebert A and Fofana M (2009). Rice yield gap due to iron toxicity in West Africa. J. Agron. Crop Sci. 195: 66-76. https:// doi.org/10.1111/j.1439-037X.2008.00339.X

Baldoni E, Mattana M, Locatelli F, Consonni R, et al. (2013). Analysis of transcript and metabolite levels in Italian rice (Oryza sativa L.) cultivars subjected to osmotic stress or benzothiadiazole treatment. Plant Physiol. Biochem. 70: 492-503. https://doi.org/10.1016/j.plaphy.2013.06.016

Banerjee A and Roychoudhury A (2015). WRKY proteins: signaling and regulation of expression during abiotic stress responses. Sci. World J. 2015: 807560. https://doi.org/10.1155/2015/807560

Bazzini AA, Almasia NI, Manacorda CA, Mongelli VC, et al. (2009). Virus infection elevates transcriptional activity of miR164a promoter in plants. BMC Plant Biol. 9: 152. https://doi.org/10.1186/1471-2229-9-152

Becker M and Asch F (2005). Iron toxicity in rice-conditions and management concepts. J. Plant Nutr. Soil Sci. 168: 558573. https://doi.org/10.1002/jpln.200520504

De Bodt S, Theissen G and Van de Peer Y (2006). Promoter analysis of MADS-box genes in eudicots through phylogenetic footprinting. Mol. Biol. Evol. 23: 1293-1303. https://doi.org/10.1093/molbev/msk016

Bustin SA, Benes V, Garson JA, Hellemans J, et al. (2009). The MIQE guidelines: minimum information for publication of quantitative real-time PCR experiments. Clin. Chem. 55: 611-622. https://doi.org/10.1373/clinchem.2008.112797

Dobermann A and Fairhurst TH (2000). Rice: nutrient disorders and nutrient management. 1st edn. Potash \& Phosphate Institute (PPI), Potash \& Phosphate Institute of Canada (PPIC) and International Rice Research Institute (IRRI).

Elec V, Quimio CA, Mendoza R, Sajise AGC, et al. (2013). Maintaining elevated Fe2+ concentration in solution culture for the development of a rapid and repeatable screening technique for iron toxicity tolerance in rice (Oryza sativa L.). Plant Soil 372: 253-264. https://doi.org/10.1007/s11104-013-1739-4

Finatto T, de Oliveira AC, Chaparro C, da Maia LC, et al. (2015). Abiotic stress and genome dynamics: specific genes and transposable elements response to iron excess in rice. Rice (N. Y.) 8: 13. https://doi.org/10.1186/s12284-015-0045-6

Jain M, Nijhawan A, Tyagi AK and Khurana JP (2006). Validation of housekeeping genes as internal control for studying gene expression in rice by quantitative real-time PCR. Biochem. Biophys. Res. Commun. 345: 646-651. https://doi.

Genetics and Molecular Research 16 (3): gmr16039694 
org/10.1016/j.bbrc.2006.04.140

Jimmy JL and Babu S (2015). Role of OsWRKY transcription factors in rice disease resistance. Trop. Plant Pathol. 40: 355-361. https://doi.org/10.1007/s40858-015-0058-0

Kobayashi T and Nishizawa NK (2012). Iron uptake, translocation, and regulation in higher plants. Annu. Rev. Plant Biol. 63: 131-152. https://doi.org/10.1146/annurev-arplant-042811-105522

Kobayashi T, Yoshihara T, Jiang T, Goto F, et al. (2003). Combined deficiency of iron and other divalent cations mitigates the symptoms of iron deficiency in tobacco plants. Physiol. Plant. 119: 400-408. https://doi.org/10.1034/j.13993054.2003.00126.x

Krzyzanowski FC, Vieira RD and Neto JBF (1999). Vigor de sementes: conceitos e testes. Associação Brasileira de Sementes, Londrina.

Lescot M, Déhais P, Thijs G, Marchal K, et al. (2002). PlantCARE, a database of plant cis-acting regulatory elements and a portal to tools for in silico analysis of promoter sequences. Nucleic Acids Res. 30: 325-327. https://doi.org/10.1093/ $\underline{\text { nar } / 30.1 .325}$

Livak KJ and Schmittgen TD (2001). Analysis of relative gene expression data using real-time quantitative PCR and the $2^{(-\Delta \Delta C(\mathrm{~T}))}$ Method. Methods 25: 402-408. https://doi.org/10.1006/meth.2001.1262

Nagajyoti PC, Lee KD and Sreekanth TVM (2010). Heavy metals, occurrence and toxicity for plants: a review. Environ. Chem. Lett. 8: 199-216. https://doi.org/10.1007/s10311-010-0297-8

Nava G and Bohnen H (2002). Oxidação de ferro em raízes de dois cultivares de arroz em solução de solo inundado. Rev. Bras. Ciênc. Solo 26: 325-332. https://doi.org/10.1590/S0100-06832002000200005

Pereira EG, Oliva MA, Rosado-Souza L, Mendes GC, et al. (2013). Iron excess affects rice photosynthesis through stomatal and non-stomatal limitations. Plant Sci. 201-202: 81-92. https://doi.org/10.1016/j.plantsci.2012.12.003

Petit JM, van Wuytswinkel O, Briat JF and Lobréaux S (2001). Characterization of an iron-dependent regulatory sequence involved in the transcriptional control of AtFerl and ZmFerl plant ferritin genes by iron. J. Biol. Chem. 276: 55845590. https://doi.org/10.1074/jbc.M005903200

Phukan UJ, Jeena GS and Shukla RK (2016). WRKY transcription factors: molecular regulation and stress responses in plants. Front. Plant Sci. 7: 760. https://doi.org/10.3389/fpls.2016.00760

Ricachenevsky FK, Sperotto RA, Menguer PK and Fett JP (2010). Identification of Fe-excess-induced genes in rice shoots reveals a WRKY transcription factor responsive to Fe, drought and senescence. Mol. Biol. Rep. 37: 3735-3745. https://doi.org/10.1007/s11033-010-0027-0

Rice WRKY Working Group (2012). Nomenclature report on rice WRKY's - Conflict regarding gene names and its solution. Rice (N. Y.) 5: 3. https://doi.org/10.1186/1939-8433-5-3

Shen C, Yang Y, Liu K, Zhang L, et al. (2016). Involvement of endogenous salicylic acid in iron-deficiency responses in Arabidopsis. J. Exp. Bot. 67: 4179-4193. https://doi.org/10.1093/jxb/erw196

da Silveira VC, de Oliveira AP, Sperotto RA, Espindola LS, et al. (2007). Influence of iron on mineral status of two rice (Oryza sativa L.) cultivars. Braz. J. Plant Physiol. 19: 127-139. https://doi.org/10.1590/S1677-04202007000200005

Stein RJ, Lopes SIG and Fett JP (2014). Iron toxicity in field-cultivated rice: contrasting tolerance mechanisms in distinct cultivars. Theor. Exp. Plant Physiol. 26: 135-146. https://doi.org/10.1007/s40626-014-0013-3

Thomine S and Vert G (2013). Iron transport in plants: better be safe than sorry. Curr. Opin. Plant Biol. 16: 322-327. https://doi.org/10.1016/j.pbi.2013.01.003

Walker EL and Connolly EL (2008). Time to pump iron: iron-deficiency-signaling mechanisms of higher plants. Curr. Opin. Plant Biol. 11: 530-535. https://doi.org/10.1016/j.pbi.2008.06.013

Wang Y, Feng L, Zhu Y, Li Y, et al. (2015). Comparative genomic analysis of the WRKY III gene family in populus, grape, arabidopsis and rice. Biol. Direct 10: 48. https://doi.org/10.1186/s13062-015-0076-3

Wu K-L, Guo Z-J, Wang HH and Li J (2005). The WRKY family of transcription factors in rice and Arabidopsis and their origins. DNA Res. 12: 9-26. https://doi.org/10.1093/dnares/12.1.9

Wu L-B, Shhadi MY, Gregorio G, Matthus E, et al. (2014). Genetic and physiological analysis of tolerance to acute iron toxicity in rice. Rice (N. Y.) 7: 8. https://doi.org/10.1186/s12284-014-0008-3

Yan JY, Li CX, Sun L, Ren JY, et al. (2016). A WRKY transcription factor regulates Fe translocation under Fe deficiency. Plant Physiol. 171: 2017-2027. https://doi.org/10.1104/pp.16.00252

Yoshida S, Forno DA, Cock JH and Gomez KA (1976). Laboratory manual for physiological studies of rice. IRRI, Los Baños.

Zhang J, Peng Y and Guo Z (2008). Constitutive expression of pathogen-inducible OsWRKY31 enhances disease resistance and affects root growth and auxin response in transgenic rice plants. Cell Res. 18: 508-521. https://doi. org/10.1038/cr.2007.104

Genetics and Molecular Research 16 (3): gmr16039694 


\section{Supplementary material}

Table S1. Differential expression in leaves of 18-day-old rice seedlings after 4 days under iron excess (Finatto et al., 2015).

Table S2. Oligonucleotides used for qRT-PCR experiment.

Table S3. Analysis of variance for shoot length (SL), root length (RL), root number (RN), leaf number (LN), coleoptile length (CL), shoot dry mass (SDM), and root dry mass (RDM) of rice seedlings after 12 days under iron excess.

Table S4. Different predicted cis-regulatory elements (CRE) found in 1-kb upstream regions of OsWRKY55-like, OsWRKY46, OsWRKY64, and OsWRKY113. Only CRE with matrix score $\geq 5$ were considered. Green represents presence of a certain CRE, while red represents its absence.

Genetics and Molecular Research 16 (3): gmr16039694 